Kong. Res. J. 2(2) : 26-29, 2015

ISSN 2349-2694

Kongunadu Arts and Science College, Coimbatore.

\title{
NANO bT CLOSED SET IN NANO TOPOLOGICAL SPACES
}

\section{Krishnaveni, K.* and M. Vigneshwaran}

\author{
Department of Mathematics, Kongunadu Arts and Science College (Autonomous), Coimbatore-641029. \\ *E.mail: krishnavenikaliswami@gmail.com
}

\section{ABSTRACT}

In this paper, we introduce a new class of set namely $\mathrm{n}$ a n o bT -closed sets in nano topological space. Wealsodiscussedsomeproperties ofnanobTclosedset.

Keywords: Nano T closed and nano bT-closed.

\section{INTRODUCTION}

Nano topological space was introduced (Lellis Thivagar and Camel Richard, 2013a, b) with respect to a subset $X$ of a universe which is defined in terms of lower and upper approximations of X. He also established certain weak forms of nano open sets such as nano $\alpha$ - open sets, nano semi- open sets and nano pre open sets. b- open sets in topological spaces was introduced and studied (Andrijevic,1996). Several properties of a new type of sets called supra T-closed set and supra Tcontinuous maps was studied (Arockiarani and Trintia Pricilla, 2011). Also a new class of bT closed set in supra topological spaces was introduced and studied (Krishnaveni and Vigneshwaran, 2013). In this paper, we introduced a new class of set called nano bT - closed sets and study its basic properties.

\section{PRELIMINARIES}

\subsection{Definition}

Let $U$ be a non-empty finite set of objects called the universe and $\mathrm{R}$ be an equivalence relation on $U$ named as the indiscernibility relation. Then $U$ is divided into disjoint equivalence classes. Elements belonging to the same equivalence class are said to be indiscernible with one another. The pair $(U, R)$ is said to be the approximation space. Let $\mathrm{X} \subseteq \mathrm{U}$.

(i) The lower approximation of $X$ with respect to $\mathrm{R}$ is the set of all objects, which can be for certainly classified as $\mathrm{X}$ with respect to $\mathrm{R}$ and it is denoted by $\mathrm{L}_{\mathrm{R}}(\mathrm{X})$. That is $\mathrm{L}_{\mathrm{R}}(\mathrm{X})={ }_{x \in U} R x: R(x) \subseteq \mathrm{X}$

, where $\mathrm{R}(\mathrm{x})$ denotes the equivalence class determined by $\mathrm{x} \epsilon \mathrm{U}$.

(ii) The upper approximation of $\mathrm{X}$ with respect to $R$ is the set of all objects, which can be possibly classified as $\mathrm{X}$ with respect to $\mathrm{R}$ and it is denoted by $\mathrm{U}_{\mathrm{R}}(\mathrm{X})$.
That is $\mathrm{U}_{\mathrm{R}}(\mathrm{X})={ }_{x \in U} R x: R x \cap \mathrm{X} \neq \phi$

(iii) The boundary region of $X$ with respect to $R$ is the set of all objects, which can be classified neither as $\mathrm{X}$ nor as not-X with respect to $\mathrm{R}$ and it is denoted by $B_{R}(X)$.

That is, $B_{R}(X)=U_{R}(X)-L_{R}(X)$.

\subsection{Property}

If $(U, R)$ is an approximation s p a c e a $\mathrm{n} X, Y \subseteq U$, then

(i) $\quad \mathrm{L}_{\mathrm{R}}(\mathrm{X}) \subseteq \mathrm{X} \subseteq \mathrm{U}_{\mathrm{R}}(\mathrm{X})$.

(ii) $\quad \mathrm{L}_{\mathrm{R}}(\phi)=\underset{\mathrm{R}}{\mathrm{U}}(\phi)=\phi$ and $\mathrm{L} \underset{\mathrm{R}}{(\mathrm{U})}=\mathrm{U} \underset{\mathrm{R}}{(\mathrm{U})}=\mathrm{U}$

(iii) $\quad U_{R}(X \cup Y)=U_{R}(X) \cup U_{R}(Y)$

(iv) $\quad U_{R}(X \cap Y)=U_{R}(X) \cap U_{R}(Y)$

(v) $\quad L_{R}(X \cup Y)=L_{R}(X) \cup L_{R}(Y)$

(vi) $\quad L_{R}(X \cap Y)=L_{R}(X) \cap L_{R}(Y)$

(vii) $\mathrm{L}_{\mathrm{R}}(\mathrm{X}) \subseteq \mathrm{L}_{\mathrm{R}}(\mathrm{Y})$ and $\mathrm{U}_{\mathrm{R}}(\mathrm{X}) \subseteq \mathrm{U}_{\mathrm{R}}(\mathrm{Y})$ whenever $\mathrm{X} \subseteq \mathrm{Y}$

(viii) $\mathrm{U}_{R}\left(\mathrm{X}^{\mathrm{c}}\right)=\left[\mathrm{L}_{\mathrm{R}}(\mathrm{X})\right]^{\mathrm{c}}$ and $\mathrm{L}_{\mathrm{R}}\left(\mathrm{X}^{\mathrm{c}}\right)=\left[\mathrm{U}_{\mathrm{R}}(\mathrm{X})\right]^{\mathrm{c}}$

(ix) $\quad U_{R} U_{R}(X)=L_{R} U_{R}(X)=U_{R}(X)$

(x) $\quad L_{R} L_{R}(X)=U_{R} L_{R}(X)=L_{R}(X)$.

\subsection{Definition}

Let $U$ be the universe, $R$ be an equivalence relation on $U$ and $\tau_{R}(X)=\left\{U, \phi, L_{R}(X), U_{R}(X), B_{R}(X)\right\}$, where $\mathrm{X} \subseteq \mathrm{U}$. Then by property $2.2, \tau{ }_{\mathrm{R}}(\mathrm{X})$ satisfies the following axioms:

(i) $\mathrm{U}$ and $\phi \in \tau_{\mathrm{R}}(\mathrm{X})$.

(ii) The union of the elements of any subcollection of $\tau_{R}(X)$ is in $\tau_{R}(X)$.

(iii) The intersection of the elements of any finite subcollection of $\tau_{R}(X)$ is in $\tau_{R}(X)$. 
That is, $\tau_{R}(X)$ is a topology on $U$ called the nano topology on $U$ with respect to $X$. We call $\left(U, \tau_{R}\right.$ $(\mathrm{X})$ ) as the nano topological space. The elements of $\tau$ ${ }_{\mathrm{R}}(\mathrm{X})$ are called as nano open sets.

\subsection{Definition}

If $\left(U, \tau_{R}(X)\right)$ is a nano topological space with respect to $X$ where $X \subseteq U$ and i f $A \subseteq U$, then the nano interior of $A$ is defined as the union of all nano open subsets of $A$ and it is denoted by Nint ( A). That is, Nint ( A) is the largest nano open subset of $A$. The nano closure of $A$ is defined as the intersection of all nano closed sets containing $A$ and it is denoted by $\operatorname{Ncl}(\mathrm{A})$.

That is, $\operatorname{Ncl}(A)$ is the smallest nano closed set containing A.

\subsection{Definition}

A subset $A$ of a topological space $(X, \tau)$ is said to be b-open

if $A \subseteq \operatorname{cl}(\operatorname{int}(A)) \cup \operatorname{int}(\operatorname{cl}(A))$. The complement of $b$ - open set is called a b closed set.

\subsection{Definition}

A set $A$ of $X$ is called generalized $b$-closed set (simply gb-closed) if bcl $(\mathrm{A}) \subseteq \mathrm{U}$ whenever $\mathrm{A} \subseteq \mathrm{U}$ and $U$ is open. The complement of generalized $b$ closed set is generalized b-open set.

\subsection{Definition}

Let $(X, \mu)$ is a supra topological spaces. A subset $A$ of $(X, \mu)$ is called $T^{\mu}$-closed set if bcl ${ }^{\mu}(A) \subseteq U$ whenever $A \subseteq U$ and $U$ is $g^{\mu}$ b - open in $(X, \mu)$. The complement of $T^{\mu}$ - closed set is called $T$

$\mu$-open set.

\subsection{Definition}

A subset $A$ of a topological space $(X, \tau)$ is called regular open if $A=\operatorname{cl}(\operatorname{int}(A))$. The complement of regular open set is called regular closed set.

\subsection{Definition}

A subset $A$ of a topological space $(X, \tau)$ is called generalized b- regular closed set if bcl (A) $\subseteq \mathrm{U}$ and whenever $\mathrm{A} \subseteq \mathrm{U}$ and $\mathrm{U}$ is regular open of $(X, \tau)$. The complement of generalized $b$ - regular closed set is called generalized b- regular open set.

\subsection{Definition}

A subset $A$ of a supra topological space (X, $\mu$ ) is called bT $\mu$-closed set (Krishnaveni and Vigneshwaran, 2013) if bcl ${ }^{\mu}(A) \subseteq U$ whenever $A \subseteq U$ and $U$ is $T^{\mu}$ - open in $(X, \mu)$.

\section{NANO bT-CLOSEDSET}

\subsection{Definition}

Let $(U, \tau R(X))$ be a nano to pologic als pa ce. A subset $A$ of $(U, \tau R(X))$ is called nano $\mathrm{T}$ - closed set if $\operatorname{Nbcl}(\mathrm{A}) \subseteq \mathrm{U}$ whenever $A \subseteq U$ and $U$ is nano gb- open in $(\mathrm{U}, \tau \mathrm{R}(\mathrm{X}))$.

\subsection{Example}

Let $\mathrm{U}=\{\mathrm{a}, \mathrm{b}, \mathrm{c}, \mathrm{d}\}$ with $\mathrm{U} / \mathrm{R}=$ $\{\{a\},\{d\},\{b, c\}\}$ and $X=\{a, c\}$. Then the nano topology $\tau_{R}(X)=\{U, \phi,\{a\},\{b, c\},\{a, b, c\}\}$. The nano $T$ closed sets are $U, \phi,\{a\},\{b\},\{c\},\{d\}$, $\{a, d\},\{b, c\},\{c, d\},\{b, c, d\},\{a, c, d\}$ and $\{a, b, d\}$.

\subsection{Definition}

Let $(U, \tau R(X))$ be a nano t o p o l o g i c a l s p a c e. A subset A of (U, $\tau R(X))$ is called nano bT - closed set if $\operatorname{Nbcl}(\mathrm{A}) \subseteq \mathrm{U}$ whenever $A \subseteq U$ and $U$ is nano $T$ - open in $(\mathrm{U}, \tau \mathrm{R}(\mathrm{X}))$. The complement of nano bT closed set is called nano bT -open set

\subsection{Example}

Let $\mathrm{U}=\{\mathrm{a}, \mathrm{b}, \mathrm{c}, \mathrm{d}\}$ with $\mathrm{U} / \mathrm{R}=$ $\{\{a\},\{d\},\{b, c\}\}$ and $X=\{a, c\}$. Then the nano topology $\tau_{R}(X)=\{U, \phi,\{a\},\{b, c\},\{a, b, c\}\}$. The nano bT closed sets are $U, \phi,\{a\},\{b\},\{c\},\{d\}$, $\{a, d\},\{b, c\},\{c, d\},\{b, c, d\},\{a, c, d\},\{a, b, d\}$ and $\{\mathrm{a}, \mathrm{b}, \mathrm{c}\}$.

\subsection{Theorem}

Every nano closed set is nano bT closed.

Proof Let $A \subseteq U$ and $U$ is nano $T$ - open setSince $A$ is nano closed then $\operatorname{Ncl}(A)=A \subseteq U$. We know that $\operatorname{Nbcl}(A) \subseteq \mathrm{N}$ cl $(A) \subseteq U$, implies $\operatorname{Nbcl}(A) \subseteq U$. Therefore A is nano bT - closed.

The converse of the above theorem need not be true as seen from the following example.

\subsection{Example}

Let $\mathrm{U}=\{\mathrm{a}, \mathrm{b}, \mathrm{c}, \mathrm{d}\}$ with $\mathrm{U} / \mathrm{R}=$ $\{\{a\},\{d\},\{b, c\}\}$ and $X=\{a, c\}$. Then the nano topology $\tau_{R}(X)=\{U, \phi,\{a\},\{b, c\},\{a, b, c\}\}$. The nano closed sets are $U, \phi,\{b, c, d\},\{a, d\}$ and $\{d\}$. 
The nano bT closed sets are $U, \phi,\{a\},\{b\},\{c\}$, $\{d\},\{a, d\},\{b, c\},\{c, d\},\{b, d\},\{b, c, d\},\{a, c, d\}$, $\{a, b, d\}$ and $\{a, b, c\}$. Here the sets $\{b\},\{a\},\{c\}$, $\{d\},\{b, c\},\{c, d\},\{a, c, d\},\{a, b, d\}$ and $\{a, b, c\}$ are nano bT closed sets but not in nano closed sets.

\subsection{Theorem}

Every nano b closed set is nano bT closed.

Proof Let $A \subseteq U$ and $U$ is nano $T$ - open set. Since $A$ is nano $b$ closed then $\operatorname{Nbcl}(A) \subseteq U$. Therefore A is nano bT - closed.

The converse of the above theorem need not be true as seen from the following example.

\subsection{Example}

Let $\mathrm{U}=\{\mathrm{a}, \mathrm{b}, \mathrm{c}, \mathrm{d}\}$ with $\mathrm{U} / \mathrm{R}=$ $\{\{a\},\{d\},\{b, c\}\}$ and $X=\{a, c\}$. Then the nano topology $\tau_{\mathrm{R}}(\mathrm{X})=\{\mathrm{U}, \phi,\{\mathrm{a}\},\{\mathrm{b}, \mathrm{c}\},\{\mathrm{a}, \mathrm{b}, \mathrm{c}\}\}$. The nano $b$ closed sets are $U, \phi,\{b, c, d\},\{a, c, d\}$, \{a,b,d\}, $\quad\{c, d\}, \quad\{b, d\}, \quad\{a, d\}$, $\{b, c\},\{a\},\{b\},\{c\}$ and $\{d\}$.The nano $b T$ closed sets are $U, \phi,\{a\},\{b\},\{c\},\{d\},\{a, d\},\{b, c\}$, $\{c, d\},\{b, c, d\},\{a, c, d\},\{a, b, d\}$ and $\{a, b, c\}$. Here the set $\{a, b, c\}$ is nano $b T$ closed sets but not in nano $b$ closed set.

\subsection{Theorem} closed set.

Every nano bT - closed set is nano gb -

Proof Let $A \subseteq U$ and $U$ is nano open set. We know that every nano open set is nano $\mathrm{T}$ - open set, then $U$ is nano T- open set. Since $A$ is nano bT -closed set, we have $\mathrm{Nbcl}(\mathrm{A}) \subseteq \mathrm{U}$. Therefore A is nano gb-closed set.

\subsection{Example}

Let $\mathrm{U}=\{\mathrm{a}, \mathrm{b}, \mathrm{c}, \mathrm{d}\}$ with $\mathrm{U} / \mathrm{R}=$ $\{\{a\},\{c\},\{b, d\}\}$ and $X=\{b, d\}$. Then the nano topology $\tau_{\mathrm{R}}(\mathrm{X})=\{\mathrm{U}, \phi,\{\mathrm{b}, \mathrm{d}\}\}$. The nano $\mathrm{gb}$ closed sets are $\mathrm{U}, \phi,\{\mathrm{b}, \mathrm{c}, \mathrm{d}\},\{\mathrm{a}, \mathrm{c}, \mathrm{d}\},\{\mathrm{a}, \mathrm{b}, \mathrm{d}\}$, $\{a, b, c\},\{c, d\},\{a, b\},\{a, c\},\{a, d\},\{b, c\},\{a\},\{b\},\{c\}$ and $\{d\}$.The nano bT closed sets are $U, \phi,\{a\}$, $\{b\},\{c\},\{d\},\{a, d\},\{b, c\},\{c, d\},\{a, b\},\{a, c\},\{a, c, d\}$ and $\{a, b, c\}$. Here the set $\{b, c, d\}$ and $\{a, b, d\}$ is nano gb closed sets but not nano bT closed set.

\subsection{Theorem} closed set.

Every $\mathrm{N}$ a n o bT- closed set is nano gbr -

Proof Let $\mathrm{A} \subseteq \mathrm{U}$ and $\mathrm{U}$ is nano regular open set. We know that every nano regular open set is nano T-open set, then $U$ is nano T-open set. Since $A$ is nano bT -closed set, we have $\operatorname{Nbcl}(\mathrm{A}) \subseteq \mathrm{U}$. Therefore A is nano gbr- closed set.

\subsection{Example}

Let $\mathrm{U}=\{\mathrm{a}, \mathrm{b}, \mathrm{c}, \mathrm{d}\}$ with $\mathrm{U} / \mathrm{R}=$ $\{\{a\},\{d\},\{b, c\}\}$ and $X=\{a, c\}$. Then the nano topology $\tau_{\mathrm{R}}(X)=\{\mathrm{U}, \phi,\{\mathrm{a}\},\{\mathrm{b}, \mathrm{c}\},\{\mathrm{a}, \mathrm{b}, \mathrm{c}\}\}$. The nano gbr closed sets are $U, \phi,\{a, b, c\},\{b, c, d\}$, $\{a, c, d\},\{a, b, d\},\{a, b\},\{a, c\}\{c, d\},\{b, d\},\{a, d\}$,

$\{b, c\},\{a\},\{b\},\{c\}$ and $\{d\}$. The nano bT closed sets are $U, \phi,\{a\},\{b\},\{c\},\{d\},\{a, d\},\{b, c\}$, $\{c, d\},\{b, c, d\},\{a, c, d\},\{a, b, d\}$ and $\{a, b, c\}$. Here the sets $\{a, b\},\{a, c\}$ and $\{b, d\}$ is nano gbr closed sets but not in nano bT closed set.

\subsection{Theorem}

The union of two nano bT - closed set is nano bT- closed set.

Proof Let A and B two nano bT - closed set. Let $A \cup B \subseteq G$, where $G$ is nano $T$ - open. Since A and B are nano bT-closed sets. Therefore $\operatorname{Nbcl}(A) \cup \mathrm{Nbcl}$ (B) $\subseteq$ G. Thus $N$ bcl $(A \cup B) \subseteq G$. Hence $A \cup B$ is Nano bT- closed set.

\subsection{Theorem}

Let $A$ be $n$ a $n$ o bT -closed set of $(U, X)$. Then Nbcl (A) - A does not contain any non empty nano T- closed set.

Proof: Necessity Let A be $\mathrm{n}$ a n o bT - closed set. suppose $\mathrm{F} \neq \phi$ is a $\mathrm{n}$ a $\mathrm{n}$ o $\mathrm{T}$ - closed set of Nbcl (A) - A. Then $F \subseteq N$ bcl (A) - A implies $F \subseteq$ $\operatorname{Nbcl}(A)$ and $A^{C}$. This implies $A \subseteq F^{C}$. Since $A$ is nano bT- closed set, $\operatorname{Nbcl}(A) \subseteq \mathrm{U}^{\mathrm{C}}$.Consequently, $\mathrm{F} \subseteq[\mathrm{Nbcl}$ $(A)]^{C}$. Hence $F \subseteq \operatorname{Nbcl}(A) \cap[\operatorname{Nbcl}(A)]=\phi$. Therefore $\mathrm{F}$ is empty, a contradition.

Sufficiency: Suppose $A \subseteq U$ and that $U$ is nano $\mathrm{T}$ - open. If $\mathrm{N}$ bcl $(\mathrm{A}) \not \subset \mathrm{U}$. Then $\operatorname{Nbcl}(\mathrm{A}) \cap \mathrm{U}^{\mathrm{C}}$ is a not empty $\mathrm{n}$ a $\mathrm{n}$ o $\mathrm{T}$ - closed subset of $\operatorname{Nbcl}(\mathrm{A})$ - A.

Hence $\mathrm{Nbcl}(\mathrm{A}) \cap \mathrm{U}^{\mathrm{C}}=\phi$ and $\mathrm{N} \operatorname{bcl}(\mathrm{A}) \subseteq$ U.Therefore $\mathrm{A}$ is nano $\mathrm{bT}$ - closed.

\subsection{Theorem}

If $\mathrm{A}$ is nano $\mathrm{bT}$ - closed set in a supra topological space $(\mathrm{U}, \mathrm{X})$ and $\mathrm{A} \subseteq \mathrm{B} \subseteq \mathrm{Nbcl}(\mathrm{A})$ then $\mathrm{B}$ is also nano bT- closed set.

Proof Let $U$ be nano T- open in set $(U, X)$ such that $\mathrm{B} \subseteq \mathrm{U}$. Since $\mathrm{A} \subseteq \mathrm{B} \Rightarrow \mathrm{A} \subseteq \mathrm{U}$ and since $\mathrm{A}$ is nano 
bT -closed set in $(\mathrm{U}, \mathrm{X}) . \operatorname{Nbcl}(\mathrm{A}) \subseteq \mathrm{U}$, since $\mathrm{B} \subseteq$ $\operatorname{Nbcl}(\mathrm{A})$. Then $\mathrm{N}$ bcl(B) $\subseteq$ U. Therefore $\mathrm{B}$ is also nano bT - closed set in $(\mathrm{U}, \mathrm{X})$

\subsection{Theorem}

Let $A$ be nano $b T$ - closed set then $A$ is nano $b$ - closed if $\mathrm{Nbcl}(\mathrm{A})-\mathrm{A}$ is nano $\mathrm{T}$ - closed.

Proof Let $A$ be nan o bT- closed set. If $A$ is nano $b$ - closed, we have $\mathrm{N}$ bcl $(\mathrm{A})$ - $\mathrm{A}=\phi$, which is nano $\mathrm{T}$ closed. Conversely, let $\mathrm{N}$ bcl(A)-A is nano bT - closed. Then by the theorem 3.13, Nbcl (A) - A does not contain any non empty nano $\mathrm{T}$ - closed and $\mathrm{N}$ bcl (A)$\mathrm{A}=\phi$. Hence $\mathrm{A}$ is nano $\mathrm{b}$ - closed.

\subsection{Theorem}

A subset $A \subseteq X$ is nano bT - open iff $F \subseteq N$ bint(A) whenever $F$ is nano $T$ - closed and $F \subseteq A$.

Proof Let $A$ be $n$ a n o bT - open set and suppose $F \subseteq A$, where $F$ is nano $T$ - closed. Then $X-A$ is nano bT - closed set contained in the $\mathrm{n}$ a $\mathrm{n}$ o $\mathrm{T}$ open set $\mathrm{X}-\mathrm{F}$. Hence $\mathrm{N}$ bcl $(\mathrm{X}-\mathrm{A}) \subseteq \mathrm{X}-\mathrm{F}$. Thus $\mathrm{F} \subseteq$ $\mathrm{N}$ bint(A). Conversely, if $\mathrm{F}$ is nano $\mathrm{T}$ - closed set with $F \subseteq \operatorname{Nbint}(A)$ and $F \subseteq A$, then $X$-Nbint $(A) \subseteq X$ $-\mathrm{F}$. This implies that $\operatorname{Nbcl}(\mathrm{X}-\mathrm{A}) \subseteq \mathrm{X}-\mathrm{F}$. Hence $\mathrm{X}-\mathrm{A}$ is nano bT - closed. Therefore A is nano bT - open set.

\subsection{Theorem}

If $\mathrm{B}$ is nano $\mathrm{T}$ - open and $\mathrm{n}$ a $\mathrm{n}$ o bT - closed set in $\mathrm{X}$, then $\mathrm{B}$ is nano $\mathrm{b}$ - closed.

Proof Since B is nano T- open and $\mathrm{n}$ a $\mathrm{n} \mathrm{o}$ bT closed then $\mathrm{Nbcl}(\mathrm{B}) \subseteq \mathrm{B}$, but $\mathrm{B} \subseteq \mathrm{N}$ bcl(B). Therefore $B=\operatorname{Nbcl}(B)$.Hence $B$ is nano $b$ - closed.

\subsection{Corollary}

If $\mathrm{B}$ is nano open and $\mathrm{n}$ a $\mathrm{n}$ o bT - closed set in X. Then B is nano b-closed.

\subsection{Theorem}

Let $\mathrm{A}$ be nano $\mathrm{g}$ b-open and nano $\mathrm{bT}$ closed set. Then $\mathrm{A} \cap \mathrm{F}$ is nanoT-closed whenever $\mathrm{F}$ is nano $\mathrm{b}$ - closed.

Proof Let $\mathrm{A}$ be nano $\mathrm{g}$ b-open and $\mathrm{n}$ a $\mathrm{n} \mathrm{o}$ bTclosed set then $\operatorname{Nbcl}(A) \subseteq A$ and also $A \subseteq \operatorname{Nbcl}(A)$. Therefore $\mathrm{N}$ bcl $(\mathrm{A})=\mathrm{A}$. Hence $\mathrm{A}$ is nano $\mathrm{b}$-closed. Since $F$ is nano $b$-closed. Therefore $A \cap F$ is nano $\mathrm{b}$ - closed in $\mathrm{X}$. Hence $\mathrm{A} \cap \mathrm{F}$ is nano $\mathrm{T}$ - closed in $\mathrm{X}$.

From the above theorem and example we have the following diagram

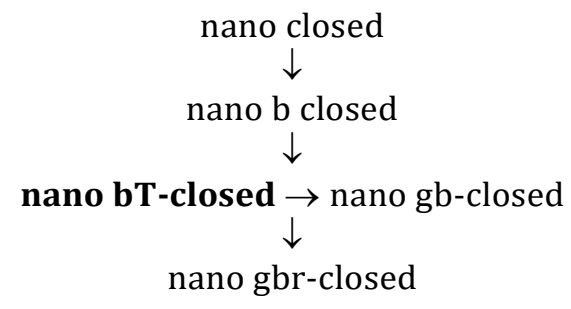

\section{REFERENCES}

Andrijevic, D. (1996). On b- open sets, mat. Vesnik 48(1-2): 59-64.

Arockiarani, I. and M. Trinita Pricilla, (2011). On supra T-closed sets, Int. J. Math. Arch., 2(8): 1376-1380.

Krishnaveni, K. and M. Vigneshwaran, (2013). On bT-Closed sets in supra topological spaces. Int. J. Math. Arch., 4(2): 1-6.

Lellis Thivagar and Camel Richard, (2013a). On nano continuity. Mathematical theory and modeling, 7: 32-37.

Lellis Thivagar and Camel Richard, (2013b). On nano forms of weakly open sets. Int. J. Math. Stat. Inv., 1(1): 31-37. 